\title{
Identification of the Risk Factors for Recurrence of Stage II Colorectal Cancer
}

\author{
Sho Sawazaki ${ }^{1)}$, Manabu Shiozawa ${ }^{1)}$, Yusuke Katayama ${ }^{1)}$, Koji Numata ${ }^{1)}$, \\ Masakatsu Numata ${ }^{1)}$, Teni Godai ${ }^{1)}$, Akio Higuchi ${ }^{1)}$, Yasushi Rino ${ }^{2)}$, \\ Munetaka Masuda ${ }^{2)}$ and Makoto Akaike ${ }^{1)}$ \\ ${ }^{1)}$ Department of Gastrointestinal Surgery, Kanagawa Cancer Center \\ ${ }^{2)}$ Department of Surgery, Yokohama City University
}

\begin{abstract}
Introduction: The use of adjuvant chemotherapy in stage II colorectal cancer patients remains controversial. However, patients with specific clinicopathological features are thought to have a high risk for recurrence. The aim of this study was to identify the subgroup of patients at the greatest risk by investigating the clinicopathological features associated with a poor survival in patients with stage II disease. Patients \& Methods: A total of 414 patients with stage II colorectal cancer who underwent curative resection between January 1990 and September 2007 at Kanagawa Cancer Center were enrolled. The clinicopathological data of the patients were retrospectively evaluated.

Results: The median follow-up period was 62.5 months. The 5-year disease-free survival rate was $89.6 \%$ in the study group as a whole. A univariate analysis of 5-year disease-free survival identified three factors: lymphatic invasion $(p=0.001)$, the preoperative serum CEA level $(>5 \mathrm{ng} / \mathrm{ml})(p=0.005)$ and the CA19-9 level ( $>37 \mathrm{U} / \mathrm{ml})(p=0.006)$. A multivariate analysis of 5 -year disease-free survival identified one independent factor: lymphatic invasion (HR: 1.89; 95\% CI: 1.02-3.50; $p=0.044$ ).

Conclusions: Patients with stage II colorectal cancer who exhibit lymphatic invasion are at a high risk for recurrence.
\end{abstract}

Received: June 13, 2013/Accepted: July 30, 2013 Correspondence to: Sho Sawazaki Department of Gastrointestinal Surgery, Kanagawa Cancer Center, 1-1 -2 Nakao, Asahi-ku, Yokohama 241-0815, Japan
Key words: colorectal cancer, stage II, high risk, disease free survival, adjuvant chemotherapy

\section{Introduction}

According to the colorectal cancer treatment guidelines ${ }^{1)}$, the administration of adjuvant chemotherapy following curative surgical resection in stage III colorectal cancer patients is recommended because it reduces the rate of tumor recurrence and improves disease-free and overall survival ${ }^{2,3)}$. However, the usefulness of postoperative adjuvant chemotherapy for treating stage II disease has not been verified $^{4-7)}$, and the administration of adjuvant chemotherapy in all patients with stage II disease has not been validated. In other guidelines, groups at a high risk for recurrence of stage II colorectal cancer have been defined, and the use of adjuvant chemotherapy in such groups is recommended. The American Society of Clinical Oncology (ASCO) guidelines ${ }^{8)}$ recommend considering the use of adjuvant chemotherapy in patients with stage II colon cancer, such as those with $<12$ regional lymph nodes examined, T4 lesions, perforation, poorly-differentiated adenocarcinoma, mucinous adenocarcinoma or signet-ring cell carcinoma. The European Society for Medical Oncology (ESMO) guidelines ${ }^{9)}$ recommend that adjuvant chemotherapy be considered in patients with the following high-risk factors: T4 lesions, poorly-differentiated adenocarcinoma/undifferentiated carcinoma, vascular invasion, lymphatic vessel invasion, perineural invasion, obstruction or perforation on initial presentation, $<12$ regional lymph nodes examined or a high carcinoembryonic antigen (CEA) level. The aim of this study was to 
Table 1 Clinicopathological features of patients with stage II colorectal cancer

\begin{tabular}{ccc}
\hline Factor & Category & Number of patients $(\mathrm{n}=414)$ \\
\hline Gender & Male & 267 \\
& Female & 147 \\
Age & median & $65(23-91)$ \\
Colon or Rectum & Colon & 302 \\
& Rectum & 112 \\
T stage & T3 & 246 \\
& T4 & 168 \\
Number of lymph node & median & 35 \\
examined & & 269 \\
Adjuvant chemotherapy & Absent & 142 \\
& Present & 369 \\
Recurrence & - & 45 \\
& + &
\end{tabular}

identify the subgroups of patients at the greatest risk by investigating the clinicopathological features associated with poor survival in patients with stage II disease.

\section{Methods}

Patients

Between January 1990 and September 2007, a total of 1,804 patients with colorectal cancer underwent curative resection at Kanagawa Cancer Center. We retrospectively reviewed the following clinicopathological data of 414 patients with stage II disease: clinical characteristics, preoperative tumor staging, details of surgery, postoperative histopathology and the results of follow-up. All patients were staged based on the findings of preoperative colonoscopy, imaging of the chest, abdomen and pelvis with computed tomography and radiographic contrast enemas. Patients with synchronous cancer of other organs or who died due to other causes were excluded.

Treatment and follow-up

All patients underwent standard curative resection at our department. The tumor location was classified into two categories: right colon (cecum, ascending colon and transverse colon) and left colon (descending colon, sigmoid colon, rectum and proctos). The patients received follow-up with computed tomography and measurement of the serum CEA and CA19-9 levels. The median follow-up period was 62.5 months. In each case, after curative surgery, the decision whether to administer adjuvant chemotherapy was left to the clinical discretion of the attending physician. A 5-fluorouracil (5-FU)-based regimen was used for adjuvant chemotherapy. The duration of treatment was at least six months.

Histopathological analysis

All resected specimens underwent standard histopathological analyses. After surgery, the disease was pathologically staged according to the $7^{\text {th }}$ UICC TNM classification ${ }^{10}$. Complete tumor resection was performed in all patients. Well-differentiated, moderately-differentiated, poorly-differentiated and mucinous adenocarcinomas were evaluated histopathologically. The presence of local and distant recurrence was confirmed clinically, radiologically and/or histopathologically.

\section{Statistical analysis}

Disease-free survival rates were calculated using the Kaplan-Meier method, and survival curves were compared with the log-rank test. A Cox regression analysis was used for the univariate and multivariate analyses. The variables that reached statistical significance $(p<0.05)$ were entered into a multivariate analysis. In all cases, $p$ values $<0.05$ were considered to indicate statistical significance. All statistical analyses were performed using the Dr. SPSS II software program, version 11.0. 1J for Windows (SPSS, Inc., Chicago, IL).

\section{Results}

The clinicopathological features of the 414 patients with stage II colorectal cancer are shown in Table 1 . Forty-five patients developed recurrence. Local recurrence was defined as intrapelvic recurrence and anastomotic recurrence. The recurrent sites includ- 
Identification of the risk factors for recurrence of stage II colorectal cancer

Table 2 Univariate analysis of prognostic factors for 5-year disease-free survival

\begin{tabular}{|c|c|c|c|c|c|}
\hline Factor & Category & $\begin{array}{c}\text { Number of patients } \\
(n=414)\end{array}$ & Hazard ratio & 95\% C.I. ${ }^{*}$ & $p$-value \\
\hline \multirow[t]{2}{*}{ Gender } & Male & 267 & 1 & & \\
\hline & Female & 147 & 0.901 & $0.485-1.675$ & N.S. ${ }^{* *}$ \\
\hline \multirow{2}{*}{ Age } & $\leqq 65$ & 208 & 1 & & \\
\hline & $>65$ & 206 & 1.262 & $0.701-2.273$ & N.S. ${ }^{* *}$ \\
\hline \multirow[t]{2}{*}{ Colon or Rectum } & Colon & 302 & 1 & & \\
\hline & Rectum & 112 & 1.706 & $0.934-3.117$ & 0.082 \\
\hline \multirow[t]{2}{*}{ Tumor location } & Right & 126 & 1 & & \\
\hline & Left & 288 & 1.370 & $0.694-2.704$ & N.S. ${ }^{* *}$ \\
\hline Tumor & $\leqq 5$ & 209 & 1 & & \\
\hline diameter(cm) & $>5$ & 204 & 0.606 & $0.331-1.106$ & N.S.** \\
\hline \multirow{2}{*}{ Pathological type } & Wel, Mod & 363 & 1 & & \\
\hline & Por, Muc & 51 & 1.348 & $0.602-3.018$ & N.S.** \\
\hline \multirow[t]{2}{*}{ T stage } & T3 & 246 & 1 & & \\
\hline & T4 & 168 & 1.456 & $0.812-2.612$ & N.S.** \\
\hline \multirow{2}{*}{$\begin{array}{l}\text { Lymphatic } \\
\text { invasion }\end{array}$} & Absent & 271 & 1 & & \\
\hline & Present & 143 & 2.460 & $1.366-4.429$ & 0.001 \\
\hline \multirow[t]{2}{*}{ Venous invasion } & Absent & 204 & 1 & & \\
\hline & Present & 210 & 1.030 & $0.574-1.848$ & N.S.** \\
\hline \multirow{2}{*}{$\begin{array}{l}\text { Number of lymph } \\
\text { node examined }\end{array}$} & $<12$ & 22 & 1 & & \\
\hline & $\geqq 12$ & 392 & 0.433 & $0.171-1.098$ & 0.078 \\
\hline Preoperative & $\leqq 5$ & 325 & 1 & & \\
\hline $\begin{array}{l}\text { serum CEA } \\
(\mathrm{ng} / \mathrm{ml})\end{array}$ & $>5$ & 81 & 2.450 & $1.303-4.606$ & 0.005 \\
\hline Preoperative & $\leqq 37$ & 347 & 1 & & \\
\hline $\begin{array}{l}\text { serum CA19-9 } \\
(\mathrm{U} / \mathrm{ml})\end{array}$ & $>37$ & 52 & 2.632 & $1.319-5.253$ & 0.006 \\
\hline Adjuvant & Absent & 269 & 1 & & \\
\hline chemotherapy & Present & 142 & 0.792 & $0.414-1.514$ & N.S.** \\
\hline
\end{tabular}

${ }^{*}$ C.I.: Confidence Interval ${ }^{* *}$ N.S. $=$ Not Significant

Table 3 Multivariate analysis of prognostic factors for 5-year survival

\begin{tabular}{cccc}
\hline Factor & Hazard ratio & $95 \%$ C.I. ${ }^{*}$ & $p$-value \\
\hline Lymphatic invasion(Present/Absent) & 1.887 & $1.018-3.496$ & 0.044 \\
$\mathrm{CEA}(>5 / \leqq 5)$ & 1.951 & $0.979-3.889$ & 0.057 \\
CA19-9 $(>37 / \leqq 37)$ & 1.910 & $0.902-4.045$ & 0.091 \\
\hline
\end{tabular}

${ }^{*}$ C.I.: Confidence Interval

ed the liver (15 patients, 29.4\%), lungs (14 patients, $27.5 \%)$, peritoneum (9 patients, $17.6 \%$ ) and local sites (9 patients, $17.6 \%)$.

The 5-year disease-free survival rate was $89.6 \%$ and the overall survival rate was $96.1 \%$. Clinicopathological features, including age, gender, tumor location, tumor diameter, pathological type, T stage, presence of lymphatic invasion, presence of venous invasion, number of lymph nodes examined, use of adjuvant chemotherapy and the preoperative serum levels of CEA and CA19-9, were analyzed. The Cox univariate regression analysis showed that the recurrence rate was significantly related to the presence of lymphatic invasion and the preoperative serum levels of CEA (>5 ng/ml) and CA19-9 (>37 U/ ml) (Table 2 ). Features such as age, gender, tumor location, tumor diameter, pathological type, $\mathrm{T}$ stage, presence of venous invasion, number of lymph nodes examined and use of adjuvant chemotherapy were not found to be significant recurrent factors. The Cox multivariate analysis showed that the presence of lymphatic invasion $(p=0.044)$ was the only independent prognostic factor significantly related to recurrence in patients with stage II colorectal cancer (Table 3 ).

The 5-year disease-free survival rates differed significantly between the patients without this prognostic factor (93.0\%) and those with this prognostic factor $(83.2 \%, p=0.0019$; Fig. 1$)$. Among the patients with this prognostic factor, the 5 -year disease-free 


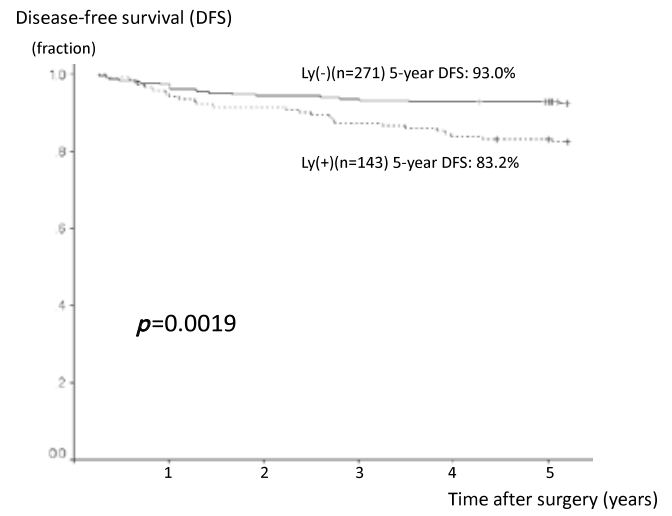

Fig. 1 Disease-free survival of patients with stage II colorectal cancer: 271 patients without lymphatic invasion, and 143 with it.

survival rates differed between the patients who received adjuvant chemotherapy (89.2\%) and those who did not $(77.6 \%, p=0.068)$, although the 5 -year overall survival rates did not differ significantly $(93.8 \%$ vs $89.5 \%, p=0.35)$.

\section{Discussion}

Many trials have shown that the administration of adjuvant chemotherapy in patients with stage III colorectal cancer is beneficial, whereas the benefits in patients with stage II disease remain controversial. In the QUASAR (Quick And Simple And Reliable) trial ${ }^{5)}$, chemotherapy with fluorouracil and folinic acid was found to improve the survival rates of patients with stage II colorectal cancer, although the absolute improvements were small. Sargent D. et al. ${ }^{11)}$ compared the use of surgery alone with surgery and FU-based adjuvant chemotherapy and found adjuvant chemotherapy to be associated with a significant improvement in 8-year overall survival (72.2\% versus $66.8 \% ; p=0.026)$. Lin BR et al. ${ }^{12)}$ compared the use of surgery alone with surgery and oral uracil and tegafur (UFT) adjuvant chemotherapy and found adjuvant chemotherapy to be associated with a significant improvement in 5-year overall survival $(89.1 \%$ versus $84.2 \%$; $p=0.018$ ).

On the other hand, a literature-based meta-analysis by an ASCO panel working in collaboration with the Cancer Care Ontario Practice Guideline Initiative ${ }^{8)}$ found no evidence of any statistically significant survival benefits for adjuvant chemotherapy in stage II patients. The authors concluded that the routine use of adjuvant chemotherapy for medically fit patients with stage II colon cancer is not recommended, although it can be considered in high-risk patients (those with inadequately sampled nodes, T4 lesions, perforation or poorly-differentiated histology). Using the Surveillance, Epidemiology and End Results (SEER) -Medicare database ${ }^{13)}$, either with or without poor prognostic features, adjuvant chemotherapy was not found to substantially improve overall survival among patients with stage II colon cancer.

In the American Joint Committee on Cancer study ${ }^{14}$, T4aN0 and T4bN0 in stage II were found to be associated with a poorer prognosis than T1-2N1a and $\mathrm{T} 1-2 \mathrm{~N} 1 \mathrm{~b}$ in stage III in terms of 5 -year relative survival rates. Therefore, administering adjuvant chemotherapy is thought to be necessary in groups at a high risk for recurrence of stage II colorectal cancer. The National Comprehensive Cancer Network $(\mathrm{NCCN})$ guidelines ${ }^{15)}$ recommend that the use of adjuvant chemotherapy be considered in patients with stage II colorectal cancer, such as those with $<12$ regional lymph nodes examined, T4 lesions, perforation, peritumoral lymphovascular involvement, poorly-differentiated adenocarcinoma or perineural invasion.

In the MOSAIC trial ${ }^{16)}$, the use of LV5FU2 in addition to oxaliplatin (FOLFOX4) was compared with LV5FU2 alone in patients with stage II disease. The probabilities of surviving to six years were $86.9 \%$ and $86.8 \%$ in the FOLFOX4 and LV5FU2 groups, respectively ( $\mathrm{HR}=1.00 ; 95 \% \mathrm{CI}, 0.70$ to $1.41 ; p=0.986$ ). In an exploratory analysis, the probabilities of OS at six years in high-risk stage II patients (at least one of the following: T4 disease, tumor perforation, bowel obstruction, poorly-differentiated tumors, venous invasion or less than 10 lymph nodes examined) were $85.0 \%$ and $83.3 \%$ in the FOLFOX 4 and LV$5 \mathrm{FU} 2$ groups, respectively ( $\mathrm{HR}=0.91 ; 95 \% \mathrm{CI}, 0.61$ to $1.36 ; p=0.648$ ). This trial did not demonstrate any effectiveness of adding oxaliplatin.

Our study showed that the presence of lymphatic invasion was an independent prognostic factor in patients with stage II colorectal cancer. We evaluated lymphatic invasion using Hematoxylin-Eosin (HE) staining. Because evaluating lymphatic invasion is frequently difficult by means of HE staining alone, the additional use of D2-40 immunostaining, which is useful for evaluating lymphatic invasion, is 
therefore considered to be essential in order to accurately evaluate colorectal cancer specimens. Developing objective criteria based $\mathrm{H}-\mathrm{E}$ staining alone may therefore be difficult. However, the use of D240 staining is not standard for most general hospitals, and its high cost and the need for a pathological analysis make its use problematical. Yet, it is not necessary to use D2-40 staining for all patients. D2-40 staining should therefore be considered when lymphatic invasion cannot be fully evaluated by some pathologists.

We identified patients with stage II colorectal cancer with lymphatic invasion as being at high risk. Adjuvant chemotherapy might offer survival benefits in such patients. However, the administration of adjuvant chemotherapy in the patients with high risk factors did not improve the 5 -year overall survival rates. In addition, our study is associated with some limitations. This study retrospectively examined the risk factors for recurrence of stage II colorectal cancer and the usefulness of adjuvant chemotherapy. The decision whether to administer adjuvant chemotherapy was not standardized in our department and was left to the clinical discretion of the attending physician. The drugs used for adjuvant chemotherapy and the duration of therapy differed. Various genetic and molecular biomarkers, including microsatellite instability (MSI), loss of heterozygosity at chromosome 18q (LOH18q) and thymidylate synthase ${ }^{17)}$, were not investigated. However, we identified the subgroup of patients at high risk by investigating the clinicopathological features of 414 patients who underwent standard curative resection at the same institution. Furthermore, this study examined many regional lymph nodes (median $\mathrm{N}=35$ ); therefore, the results are credible. Considering the usefulness of various genetic and molecular biomarkers, adjuvant chemotherapy should be aggressively administered to stage II colorectal cancer patients with lymphatic invasion.

\section{References}

1) Japanese Society for Cancer of the Colon and Rectum : Colorectal Cancer Treatment Guidelines. $7^{\text {th }}$ ed., Kanehara\&Co., Tokyo, 2010

2) Moertel CG, Fleming TR, Macdonald JS, et al : Levamisole and fluorouracil for adjuvant therapy of resected colon carcinoma. N Engl J Med 322:352358,1990

3) Andre T, Colin P, Louvet C, et al : Semimonthly ver- sus monthly regimen of fluorouracil and leucovorin administered for 24 or 36 weeks as adjuvant therapy in stage II and III colon cancer : results of a randomized trial. J Clin Oncol $21: 2896-2903,2003$

4) Efficacy of adjuvant fluorouracil and folinic acid in $\mathrm{B} 2$ colon cancer. International Multicentre Pooled Analysis of B2 Colon Cancer Trials (IMPACT B2) Investigators. J Clin Oncol $17: 1356-1363,1999$

5) Gray R, Barnwell J, McConkey C, et al: Adjuvant chemotherapy versus observation in patients with colorectal cancer: a randomised study. Lancet $370: 2020-2029,2007$

6) Kopetz S, Freitas D, Calabrich AF, et al : Adjuvant chemotherapy for stage II colon cancer. Oncology (Williston Park) 22:260-270; discussion 270, 273, 275,2008

7) Rousseau B, Chibaudel B, Bachet JB, et al : Stage II and stage III colon cancer: treatment advances and future directions. Cancer J 16:202-209, 2010

8) Benson AB 3rd, Schrag D, Somerfield MR, et al: American Society of Clinical Oncology recommendations on adjuvant chemotherapy for stage II colon cancer. J Clin Oncol 22:3408-3419, 2004

9) Van Cutsem E, Oliveira J : Primary colon cancer: ESMO clinical recommendations for diagnosis, adjuvant treatment and follow-up. Ann Oncol 20(suppl4) : 49-50, 2009

10) Sobin $\mathrm{LH}$ GM, Wittekind $\mathrm{Ch}$, eds : International Union Against Cancer (UICC) TNM Classification of Malignant Tumors. Wiley-Blackwell, Oxford, 2009

11) Sargent D, Sobrero A, Grothey A, et al : Evidence for cure by adjuvant therapy in colon cancer: observations based on individual patient data from 20,898 patients on 18 randomized trials. J Clin Oncol $27: 872-877,2009$

12) Lin BR, Lai HS, Chang TC, et al : Long-term survival results of surgery alone versus surgery plus UFT (Uracil and Tegafur)-based adjuvant therapy in patients with stage II colon cancer. J Gastrointest Surg $15: 2239-2245,2011$

13) O'Connor ES, Greenblatt DY, LoConte NK, et al : Adjuvant chemotherapy for stage II colon cancer with poor prognostic features. J Clin Oncol $29: 3381-3388,2011$

14) Gunderson LL, Jessup JM, Sargent DJ, et al : Revised tumor and node categorization for rectal cancer based on surveillance, epidemiology, and end results and rectal pooled analysis outcomes. J Clin Oncol $28: 256-263,2010$

15) NCCN : NCCN Clinical Practice Guidelines in Oncology (NCCN Guidelines) Colon Cancer. version 3,292. www.nccn.org/professionals/physician_gls/pdf/colon. pdf, 2012/12/25 reference.

16) Andre T, Boni $C$, Navarro $M$, et al : Improved overall survival with oxaliplatin, fluorouracil, and leucovorin as adjuvant treatment in stage II or III colon cancer in the MOSAIC trial. J Clin Oncol $27: 3109-$ 3116, 2009

17) Akiyoshi $T$, Kobunai $T$, Watanabe $T$ : Recent approaches to identifying biomarkers for high-risk stage II colon cancer. Surg Today 42:1037-1045, 2012 